\title{
Subliminal Calibration for Machine Operation
}

\author{
Hiroshi Igarashi \\ Tokyo Denki University \\ Japan
}

\section{Introduction}

Although advances in computer, network and mechanical technologies promise practical applications of intelligent robots, there are still issues to be realizing available intelligence like a human. For example, global perspective recognition, long-term prediction, and experience-based intuition, which are typical human abilities, are difficult to implement in artificial systems using current technologies yet.

To overcome this problem, there are roughly two approaches. One is studies on artificial intelligence to make robots more intelligent like human beings. The other is to utilize human ability by human-machine cooperation. This paper focuses on the later.

A lot of studies on human-machine systems have been proposed. Teleoperation systems are typical application expected to improve the performance utilizing human abilities. For instance, master-slave systems provide realistic information mainly through force feedback to the operator (Horiguchi \& Sawaragi, 1999) (Forsyth \& Maclean, 2006). (Katsura \& Ohnishi, 2007). The systems can give initiative of machine motion to human operator. However, due to constraints on machine workspace, their applications are limited to apply them to tasks fully utilizing human abilities, e.g., a situation in which unexpected disturbances or environmental changes are occurred.

Zheng et al. proposed robot teleoperation system with a mobile in disaster sites (Zheng et al., 2004). The operator's role is especially finding victims through robot vision and they focused on assisting the recognition of the victims. For mobile robot operation, teleoperation systems to assist by autonomous behaviour of the robot in response to command input are proposed (Wang \& Liu, 2004) (Cheng et al., 1997). Most of these assists are based on designer's subjectivity such as giving repulsive force from obstacles, attraction from an optimum trajectory and so on. These assists were effective in a specific task.

However, such assists with external input force include two problems, namely; (i) human abilities could not be utilize, and (ii) hindering human learning ability. In description (i), the assumption that the robot knows its optimum motion could reduce the need for human involvement. Therefore, users must keep the initiative to utilize the human abilities. Furthermore, about description (ii), due to robot behaviour without operators' intention, such assists make operators not only confuse, but also hinder human learning abilities. Human learning ability is demonstrated by modifying his/her internal model of the machine motion so that the internal model closes to operated machine dynamics unconsciously (Yamada \& Yamaguchi, 2004). 
Proposed technique attempts to improve above two problems. In order to give an operators initiative, assist with external force by autonomous behaviour is discarded. Therefore, the machine dynamics is modified to close to operator's internal model. To estimate difference between the internal model and the machine dynamics, target tracking task, e.g. line trace, is carried out as a calibration. We expect if the internal model closed to the machine dynamics enough, the tracking error could be reduced. After the calibration, the machine has dynamics similar to the internal model and the operator could operate the machine at will.

To not hinder the human learning ability, the machine dynamics is modified without human awareness, namely "subliminal". According to cognitive science knowledge, how change of stimulus is required to notice it is quantified by "Just Noticeable Difference: JND". By considering JND, the calibration can be carried out subliminally.

Furthermore, the subliminal calibration can provide enhancement of human learning process because human learning is also to modify the internal model approaching to the operated machine dynamics (Flanagan et al., 1999). The calibration, therefore, gives high operability with short time. The subliminal calibration is implemented to vehicle operation constructed in 3D computer graphics and is verified the validity by some experiments.

This chapter is organized as follows; in the next section, a basic concept of the subliminal calibration including definition of the best operability is described. In section 3, JND by cognitive science knowledge is discussed. Next, mathematical theory of the subliminal calibration is stated in section 4 . Then, in section 5, experiments and their results are presented, and finally, this article is concluded in section 6.

\section{Basic Concept of Subliminal Calibration}

\subsection{Human initiative in human-machine systems}

One of advantages in a human-machine system is that human abilities are utilized in the system behaviour. The abilities include global perspective environmental recognition, experience-based prediction, long-term planning, and so on. Therefore, a suitable humanmachine system should give an operator initiative to utilize the abilities.

In order to improve the operation performance in human-machine systems, in conventional researches, most of assists involves addition of external forces to human command input based on the autonomous behaviour of the system. Such external force relies on the system designer's subjectivity, e.g. repulsive force from an obstacle. This may useful for safety in a particular task, but the assists cannot have versatility, that is, it works depending on designer's assumption. Furthermore, the assist may deprive the operator initiative by operator's unexpected motion of the system.

Further, such assist cannot consider human learning dynamics. In special, human skill can be improved through practices. A new system adapts to operator's skill, called "Human Adaptive Mechatronics: HAM" was proposed (Furuta, K., 2003)(Suzuki, S., 2005). In the HAM studies, evaluation and quantification of human skills are especially focused on. We proposed a technique that improves machine operability by taking into account human learning dynamics without adding external force to command input. 


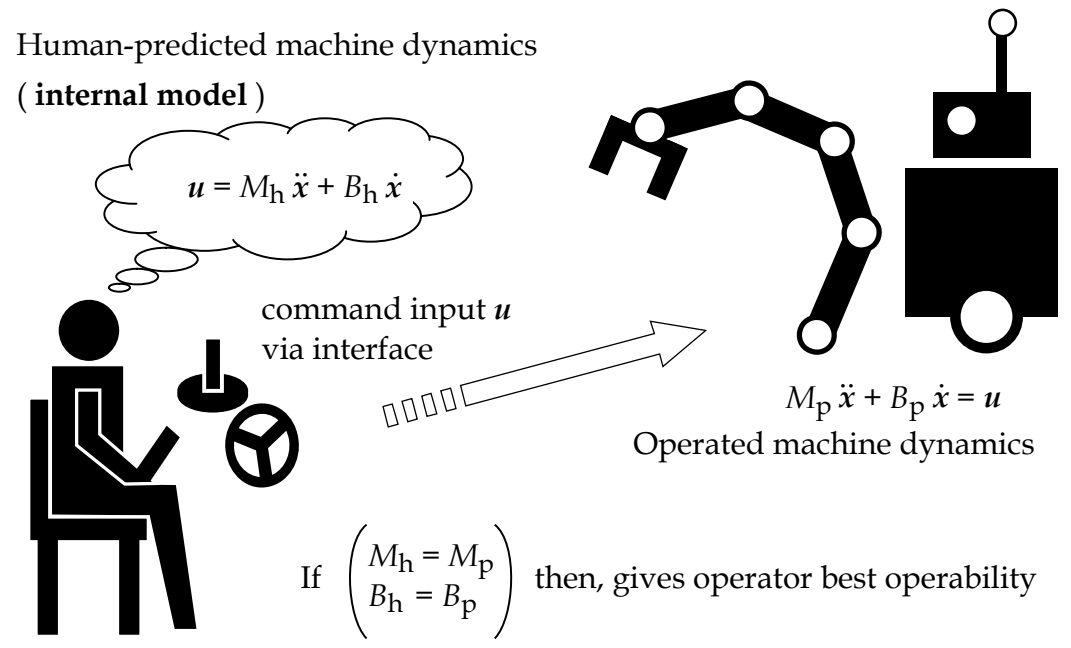

Fig. 1. Definitions of the best operability

\subsection{Discussion about the best operability}

Here, the best operability on machine operation systems is discussed. We defined the best operability is what a human operates the system at will. According to neural science knowledge, a human constructs in his/her brain a dynamics model of operated machine, called an internal model. As getting skill, the operator brushes up the internal model closing to the machine dynamics.

As shown in Fig. 1, if the internal model and the machine dynamics represented as machine impedance were closed to each enough, the system could give an operator the best operability. The idea of a new assist is the machine impedances modify to approaching the internal model. This is as if the machine learns the internal model like human beings.

Although the internal model should be obtained to give the assist technique during the operation, it seems to be impossible. Therefore, we implement the technique as a calibration in target tracking tasks, e.g. line trace by operated vehicle. We assume difference between the internal model and operated machine dynamics correlate with following error in the tracking task. After finishing the calibration, the machine would change the dynamics similar to the internal model, and then, the operator could operate it with high operability.

\subsection{Basic concept of subliminal calibration}

In our previous work, the calibration was experimented in mobile vehicle operation $(\mathrm{H}$. Igarashi, 2006). The calibration, however, could not work well for the operability. One of the reasons is confusion to changing the machine behaviour according to subject's opinions. 


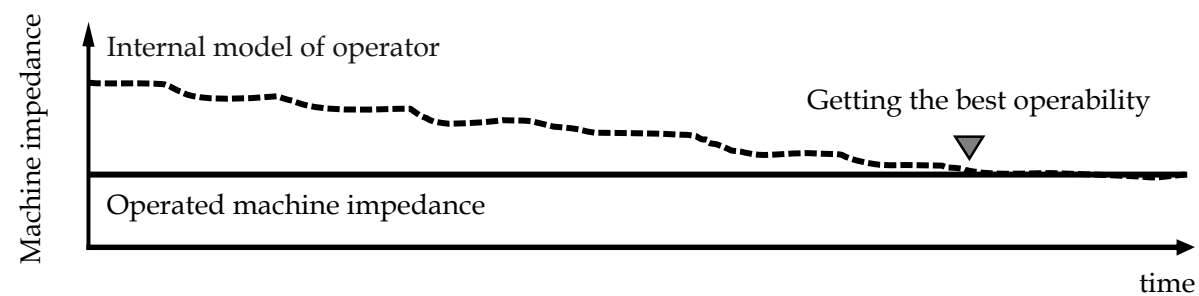

(a) Machine operation learning process without calibration: required long time.

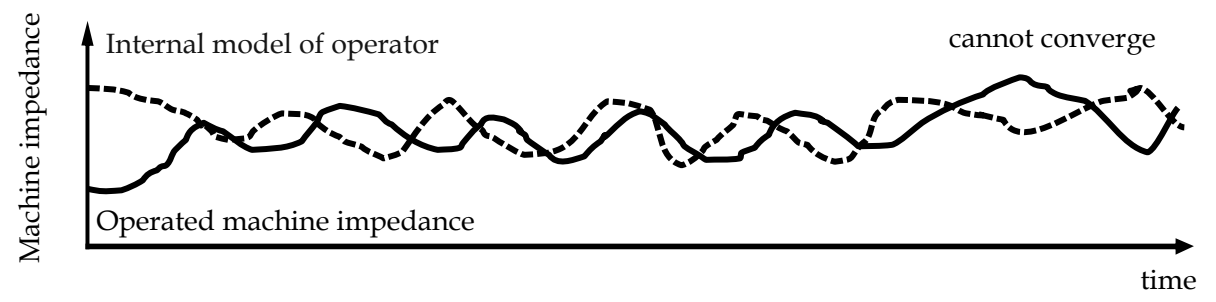

(b) Calibration by approaching human internal model without subliminal.

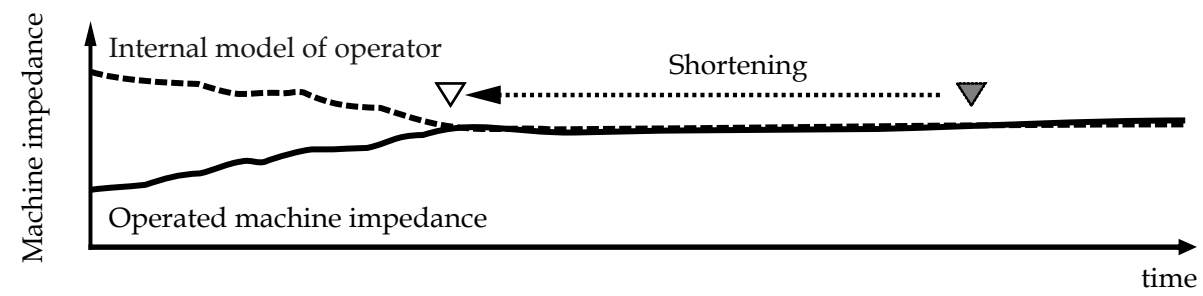

(c) Proposed subliminal calibration.

Fig. 2. Concept of proposed calibration considering human learning dynamics

This phenomenon is explained by human learning characteristics as mentioned above. That is, in spite that the operator tried to modify the internal model as shown in Fig. 2 (a), the reference model, which is represented by the machine impedances, was changed. In that case, the calibration performance was worse rather than without calibration as shown in Fig. 2 (a). Thus, the failure gave us a new suggestion that if an operator were not aware the change of machine impedances, that is "subliminal", the learning time to get the best operability could be shorten as shown in Fig. 2 (c). About human perception characteristics, Just Noticeable 
Difference: JND is investigated in cognitive science field (Teghtsoonian, 1971). In the following section, the JND to give the calibration subliminally is discussed.

\section{JND for subliminal calibration}

\subsection{General mathematical model of operated Machine}

First, for JND discussion, a simple model of operated machine in a tracking task is assumed. A motion equation of the machine with its position $x$ and input $u$, is as follows;

$$
\ddot{x}[k]=M[k]^{-1}(-B[k] \dot{x}[k]+u[k]),
$$

where, $M[k]$ and $B[k]$ denote inertia and viscous of operated machine in time step $k$, respectively. These machien impedances could be variable and the calibration modifies these to approach to the human internal model. For simplicity, these are also represented as a vector;

$$
\boldsymbol{\sigma}[k]=[M[k] \quad B[k]]^{\mathrm{T}} .
$$

A following error $e[k]$ in the following task as line tracing by vehicle driving in experiments is with a target position $x_{\mathrm{r}}$ as follows;

$$
e[k]=x_{r}[k]-x[k] .
$$

We assume if the human internal model were enough close to operated machine impedance, the following error $e$ could be reduced. Then, The subliminal calibration attempts to reduce $e[k+1]$ with modifying $\sigma[\mathrm{k}]$.

\subsection{Just Noticeable Difference}

The JND is described that a ratio between required difference to notice the stimulus $\Delta I$ and an original stimulus $I$ is approximately constant as follows;

$$
\frac{|\Delta I|}{I} \approx c \text { (constant) }
$$

where, a constant ratio $c$ is called JND or Weber's ratio and its value depends on the kind of stimulus. For instance, that JND of pressure is 0.143 and 0.079 is for brightness was reported (Teghtsoonian, 1971). B. R. Brewer et al. applied the JND to a robotic rehabilitation system for effective rehabilitation, and investigated the JND of force and position for young and elderly subjects (Brewer at el., 2005). In our previous work, the JND for notice a difference of window size is investigated for an alert system on GUI (Igarashi et al., 2005).

Then, we consider the JND of the machine impedances is described for calibration without human awareness as following equations; 


$$
\begin{gathered}
c_{M}=\frac{|M[k]-M[k-1]|}{M[k-1]}, \\
c_{B}=\frac{|B[k]-B[k-1]|}{B[k-1]},
\end{gathered}
$$

where, $c_{M}$ and $c_{B}$ denote JND for inertia and viscous, respectively. Hence, maximum variations the machine impedances to not notice their changes, $\Delta M_{\max }$ and $\Delta B_{\max }$, are described as follows;

$$
\begin{gathered}
\Delta M_{\max }[k]=\left\{\begin{array}{rc}
c_{M} M[k-1] & \text { if }(M[k]-M[k-1]) \geq 0, \\
-c_{M} M[k-1] & \text { otherwise }
\end{array},\right. \\
\Delta B_{\max }[k]=\left\{\begin{array}{cc}
c_{B} B[k-1] & \text { if }(B[k]-B[k-1]) \geq 0 \\
-c_{B} B[k-1] & \text { otherwise }
\end{array} .\right.
\end{gathered}
$$

Thus, the variations of the machine impedances, $\Delta M[\mathrm{k}]$ and $\Delta B[\mathrm{k}]$, constraints for the subliminal calibration as following equation;

$$
\begin{aligned}
& \Delta M[k]<M_{\max }[k], \\
& \Delta B[k]<B_{\max }[k],
\end{aligned}
$$

then,

$$
\boldsymbol{\sigma}[k+1]=\boldsymbol{\sigma}[k]+\Delta \boldsymbol{\sigma}[k]=\left[\begin{array}{c}
M[k]+\Delta M[k] \\
B[k]+\Delta B[k]
\end{array}\right] .
$$

If the machine impedance modification were satisfied the Eqs. (9) and (10), the operator could not notice it. Finally, the subliminal impedance modification is implemented to the calibration with JND $c$ by following filter;

$$
f_{\mathrm{JND}}(\Delta X[k], c)=\left\{\begin{array}{cc}
c X[k-1] & X[k]>c X[k-1] \\
-c X[k-1] & X[k]<-c X[k-1] \\
X[k-1] & \text { otherwise }
\end{array}\right.
$$

By applying this filter, the calibration with impedance modification can be conducted without operator awareness.

\section{Theory of subliminal calibration}

The subliminal calibration is approaching operated machine impedance to the internal model without awareness. We assume if both models were enough closed, following error 
in the tracking task could be reduced. In this section, theory of the calibration and its procedure are described.

\subsection{Human Input Model by Neural Network}

For modifying the machine impedance $\Delta \sigma[k]$ to reduce the following error $e[k+1]$, human input $u[k]$ is necessary to be predicted. The human input is predicted by the Neural Network. Input elements of the human model, $\boldsymbol{I}_{N N}[k]$, is represented as follows;

$$
\boldsymbol{I}_{N N}[k]=\left[e[k] \quad \cdots \quad e\left[k-N_{e}\right] \quad u[k] \quad \cdots \quad u\left[k-N_{u}\right]\right],
$$

where, $N_{\mathrm{e}}$ and $N_{\mathrm{u}}$ denote the number of tracing steps of the following errors $e$ and input $u$, respectively. Thus, predicted input $\hat{u}[k+1]$ is estimated as;

$$
\hat{u}[k+1]=f_{N N}\left(\boldsymbol{I}_{N N}[k]\right),
$$

where, $f_{N N}(\bullet)$ represents a forwarding Neural Network which is carried out back propergation learning with $\boldsymbol{I}_{N N}[\mathrm{k}-1]$ and its teaching signal $u[\mathrm{k}]$ in real-time.

\subsection{Subliminal modification of machine impedances}

Variation of the machine impedances $\Delta \sigma$ to reduce following error $e$ is defined here.

First, an evaluation function of following performance $J[k]$ using predicted input is calculated as follows;

$$
J[k]=\sum_{i=k}^{k+T_{p}}\left(\mu_{e} \hat{e}[i]^{2}+\mu_{0}|\sigma[0]-\sigma[i]|^{2}\right.
$$

where,

$$
\begin{gathered}
\hat{e}[i]:=\left(x_{r}-\hat{x}[i]\right), \\
\dot{\hat{x}}[k]:=\dot{x}[k], \\
\hat{x}[k]:=x[k], \\
\ddot{\hat{x}}[i]=M[i]^{-1}(-B[i] \hat{\hat{x}}[i]+\hat{u}[i]) \quad i=k, \cdots, k+T_{p} .
\end{gathered}
$$

The evaluation value $J[k]$ is determined with estimated motion of the machine until $T_{\mathrm{p}}$ step later by using predicted input $\hat{u}[k]$, and $T_{\mathrm{p}}$ denotes time step number for predictive virtual motion. Then, $\mu_{\mathrm{e}}$ is weights for the evaluation, and $\mu_{0}$ represents a weighting coefficients to constrain of impedance parameter divergence with initial impedance $\sigma[0]$.

Finally, the variation of impedance $\Delta \sigma[k]$ by steepest descent method with JND filtering in Eq. (12) is as follows;

$$
\Delta \boldsymbol{\sigma}[k]=-f_{J N D}\left(\frac{\partial J[k]}{\partial \boldsymbol{\sigma}}, \boldsymbol{c}\right)
$$


where,

$$
c:=\left[\begin{array}{l}
c_{M} \\
c_{B}
\end{array}\right] .
$$

By Eq. (20), a calibration without human awareness is realized for machine operation system. After the subliminal calibration, the impedances $\sigma[\mathrm{k}]$ are fixed to give high operability to the operator.

Note that, human would operate the machine at will soon even if differences between the internal model and the machine impedance $\sigma[k]$ are remains because human has a learning ability as swhon in Fig 2 (a). In other words, this technique enhances the human learing dynamics rather than operation performance.

\section{Experiments and Results}

Vehicle driving with the subliminal calibration is experimented (see Fig. 3). The vehicle model is based on hovercraft model because of difficult to drive and required getting skill for high performance.

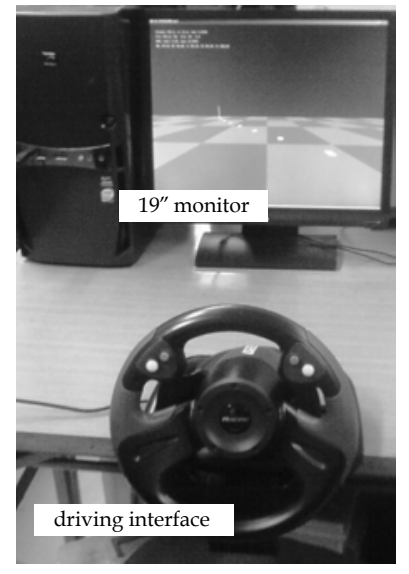

(a) Experiment setup.

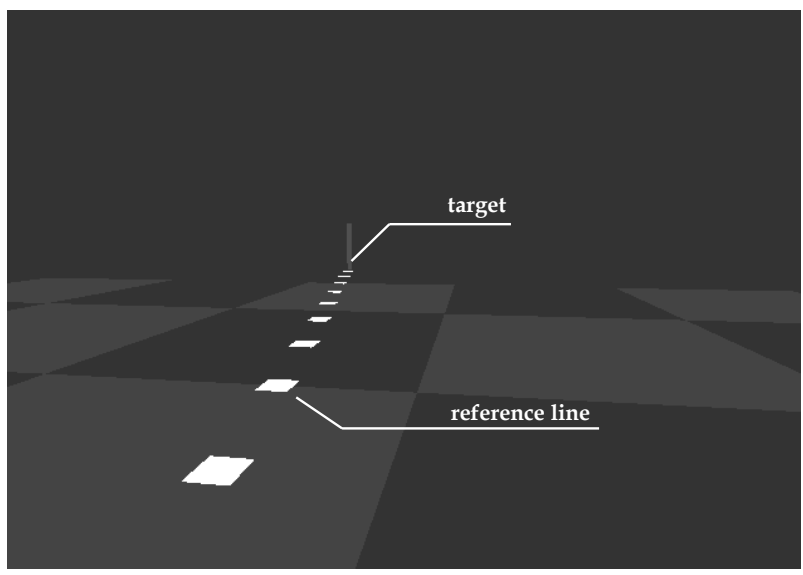

(b) Operator view in line tracking experiment.

Fig. 3. View of experimental environment in line trace task 


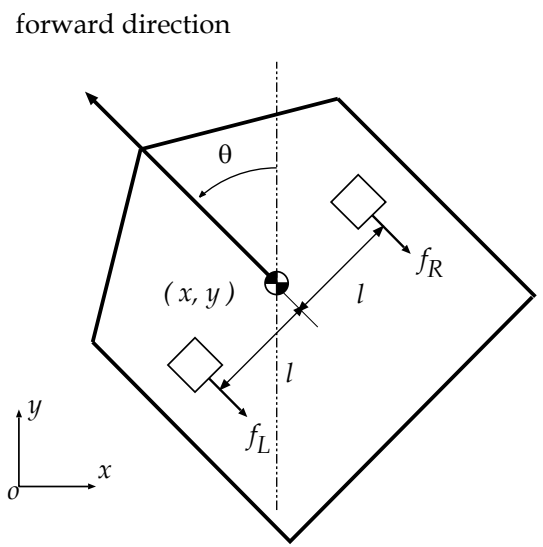

Fig. 4. Model of operated vechile in the experiment

\subsection{Operated vehicle model}

The equations of motion for an operated vehicle, which is with hovercraft-normative dynamics as shown Fig. 3 , is described here. Where $(x, y)$ denotes the global positioning axes of the vehicle, and $\theta$ the angle to forwarding direction.

Equations of motion are expressedas as follows, where $M[k]$ denotes mass, $B[k]$ translational viscosity, $I[k]$ inertial moment, and $N[k]$ rotational viscosity:

$$
\begin{gathered}
\ddot{x}[k]=\frac{1}{M[k]}\left\{-B[k] \dot{x}[k]+\cos \theta[k]\left(f_{L}[k]+f_{R}[k]\right)\right\}, \\
\ddot{y}[k]=\frac{1}{M[k]}\left\{-B[k] \dot{y}[k]+\sin \theta[k]\left(f_{L}[k]+f_{R}[k]\right)\right\}, \\
\ddot{\theta}[k]=-\frac{N[k]}{I[k]} \dot{\theta}[k]+\frac{l}{4 I[k]}\left(f_{L}[k]-f_{R}[k]\right) .
\end{gathered}
$$

Where, $f_{L}[\mathrm{k}]$ and $f_{R}[\mathrm{k}]$ denote output of thrusters as;

$$
\begin{aligned}
& f_{L}[k]:=\alpha u_{a}[k]\left(1-u_{s}[k]\right), \\
& f_{R}[k]:=\alpha u_{a}[k]\left(1+u_{s}[k]\right) .
\end{aligned}
$$

where, $u_{\mathrm{a}}[\mathrm{k}]\left(-1 \leq u_{\mathrm{a}}[\mathrm{k}] \leq 1\right)$ denotes command input for forward movement, $u_{\mathrm{s}}[\mathrm{k}]\left(-1 \leq u_{\mathrm{s}}\right.$ $[\mathrm{k}] \leq 1)$ rotation, $l$ distance between thrusters, and $\alpha$ a constant.

The subliminal calibration modifies $M, B, I$, and $N$ closer to the internal model by reducing following error. The machine impedances are rewriten Eq. (12) into following vector;

$$
\sigma[k]=\left[\begin{array}{llll}
M[k] & B[k] & I[k] & N[k]
\end{array}\right]^{T} .
$$




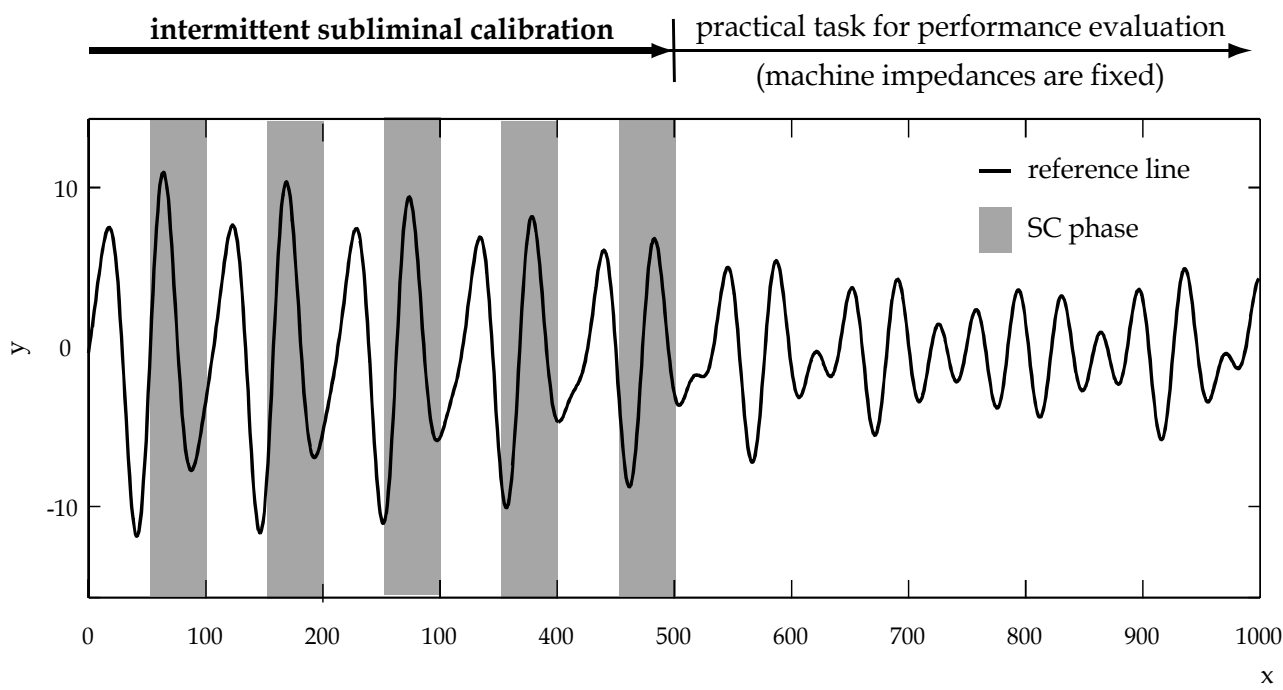

Fig. 5. Driving course and area of giving the Subliminal Calibration (SC) in the experiment

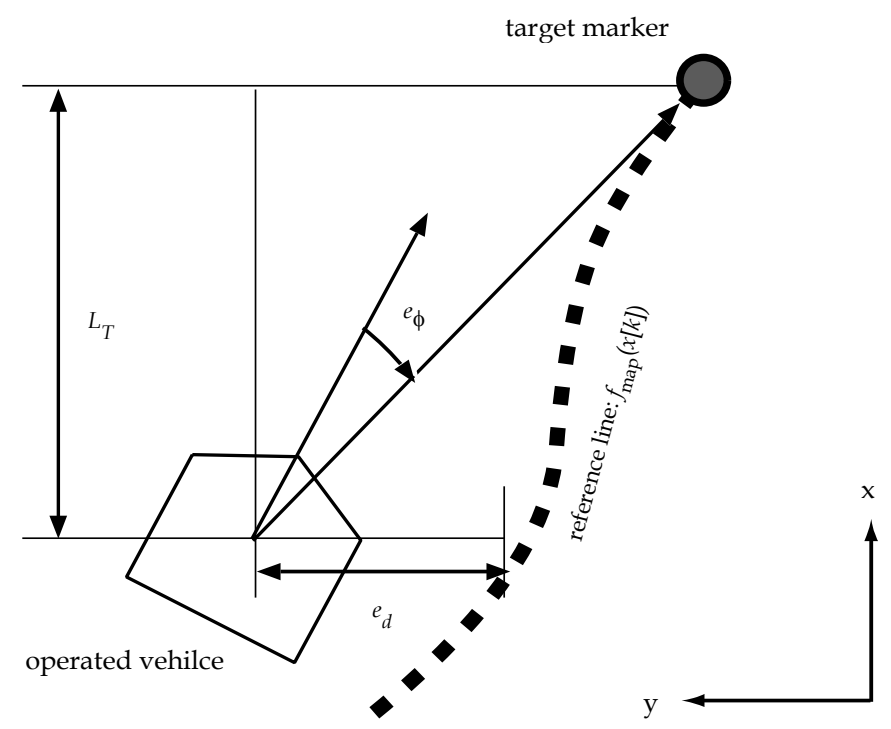

Fig. 6. Evaluation indexes for vehicle operation experiment

Similar to Eqs. (5) and (6), $c_{M}, c_{B}, c_{I}$ and $c_{N}$ are represented as JNDs on $M, B, I$, and $N$, respectively. According to our prior experimental results, $c_{M}=c_{B}=c_{I}=c_{N}=0.05$ are small enough for subliminal calibration for the vehicle operation. 
As shown in Fig. 4, participants use a driving interface to operate the vehicle in the OpenGL $3 \mathrm{D}-\mathrm{CG}$ environment. In this experiment, the accelerator was set at a constant $u_{\mathrm{a}}[\mathrm{k}]=1.0$ to focus on sterring performance.

\subsection{Experimental Setup}

The line tracking task was to have participants maneuver a vehicle through a commercial driving interface (R220, Saitek Ltd.) while observing robot camera views displayed on a 19inch monitor. Participants were instructed to follow the reference line as closely as possible by steering only with accelerator input set constant. The driving performance, with/ without calibration, for 8 participants was evaluated. To reduce effects on unconscious learning, subjects had breaks between two trials, and experiments were conducted in a random sequence.

To prevent participants from memorizing the reference line, the line generation was expressed as a combination of sine waves shown in Fig. 5;

$$
f_{\text {map }}(x)=5 \sin 0.11 x+4 \sin 0.07 x+3 \sin 0.05 x \text {. }
$$

The goal of the course is set at $x=1000$ and it takes about three minutes to finish. In this task, the subliminal calibration was set for $x<500$ and performances with/without it was compared. The calibration was conducted intermittently as shown in Fig. 6, to let participants display adaptability and learning unconscioully.

Performance evaluation indexes of the line tracing are shown in Fig. 6, following error on the $\mathrm{Y}$-axis from the reference line is expressed by $e_{\mathrm{d}}$ and those in orientation between the target and the the vehicle, by $e_{\phi}$, then we obtain the following evaluation values:

$$
\begin{gathered}
e_{\mathrm{d}}[k]=y[k]-f_{\text {map }}(x[k]), \\
e_{\phi}[k]=\tan ^{-1} \frac{f_{\text {map }}\left(x[k]+L_{T}\right)-y[k]}{L_{T}}-\theta[k],
\end{gathered}
$$

Where, $L_{T}$ denotes distance on the x-axis from the vehicle to a target flag, which moves on reference line; with $L_{T}=10$ in the experiment.

The human model is constructed based on these evaluation values, namely input vector to the Nerural Network in Eq. (13) is rewriten as follows;

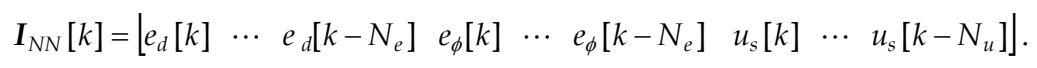

Where, in the experiments, $N_{e}=N_{u}=5$.

Because accelalation input $u_{a}=1.0$, human input is only predicted for sterring input $u_{s}$. Therefore, using forwarding Neural Network $f_{N N}$ with input $\boldsymbol{I}_{N N}[\mathrm{k}]$ in Eq. (30), the input prediction is as;

$$
\hat{u}_{s}[k+1]=f_{N N}\left(\boldsymbol{I}_{N N}[k]\right) .
$$


Then, the modification of the impedance is as follows;

$$
J[k]=\sum_{i=k}^{k+T_{p}}\left(\mu_{d} \hat{e}_{d}[i]^{2}+\mu_{\phi} \hat{e}_{\phi}[i]^{2}+\mu_{0}|\sigma[0]-\sigma[i]|^{2},\right.
$$

wehre, the initial value of the machine impedance was set to $\sigma 0]=[50,50,50,50]^{T}$. In experiments with the calibration, initial $\sigma$ would be modified to minimize $J[k]$ by Eq. (20).

\subsection{Experimental Results}

Fig. 7 shows averages of line tracking performance for all 8 participants. All participants couuld NOT notice changes of the machine impedance by the subliminal calibration through the experiment. Fig. 7 (a), (b), (c) and (d) show line following evaluation without the subliminal calibration. Note that, inspete of without the calibation, following errors decreased as $x$ increased, apparently representing improvements due to operator learning dynamics. This indicates that participants can have their internal model approach operated vehicle dynamics even without the calibration. Fig. 7 (e), (f), (g) and (h) show results of giving subliminal calibration intermittently in $x<500$.

By Fig. (e) and (g) following error $e_{\mathrm{d}}$ and $e_{\phi}$ improved over the case without the calibration, and standard deviation also improved as shown in Fig. 7(f) and (h). Note that machine impedances were intermittently updated in calibration of $x<500$ but no updating was done for $x>500$. This suggests that the calibration in the first half of the course has transformed the machine whoes impedance into the internal model and the operator could get high operability.

Next, Fig. 8 shows typical two participants who are the most skilled and unskilled operator are focused on. According to Fig. 8 (a), the skilled operator is enough high performance without the calibration. Note Fig. 8 (b) that, the subliminal calibration did not interfere in the skilled operator.

Fig. 8 (c) and (d) shows the following performance of an unskilled operator. As shown in Fig. 8 (d), even after the calibration $x>500$, the unskilled operator could keep performance, that is, the machine impedance could approach to the internal model of the unskilled operator. Therefore, the subliminal calibration can be applied without concern of operator's skill. 


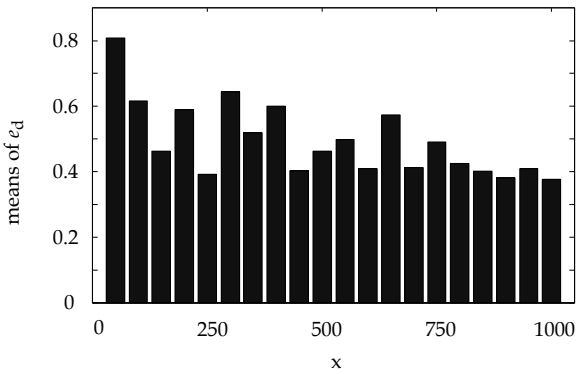

(a) means of following error $e_{\mathrm{d}}$ without $\mathrm{SC}$

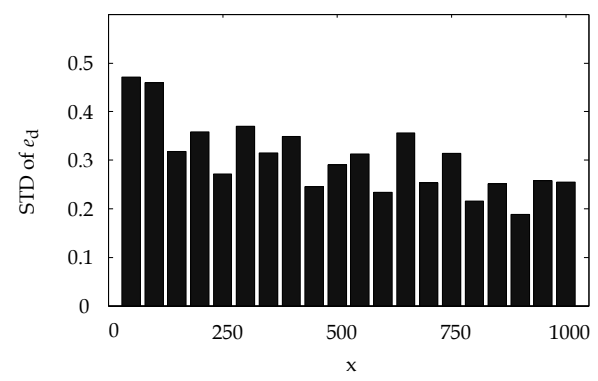

(b) STD of following error $e_{\mathrm{d}}$ without SC

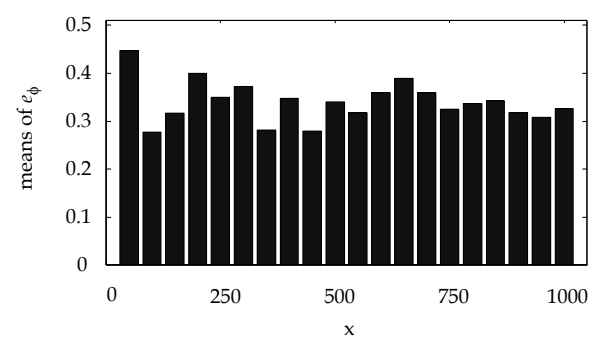

(c) means of rotational error $e_{\phi}$ without SC

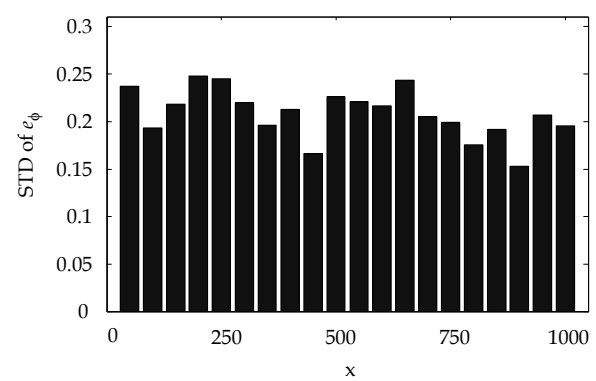

(d) STD of rotational error $e_{\phi}$ without SC

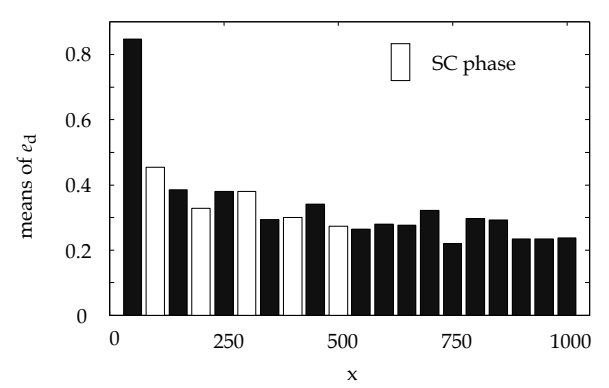

(e) means of following error $e_{\mathrm{d}}$ with $\mathrm{SC}$

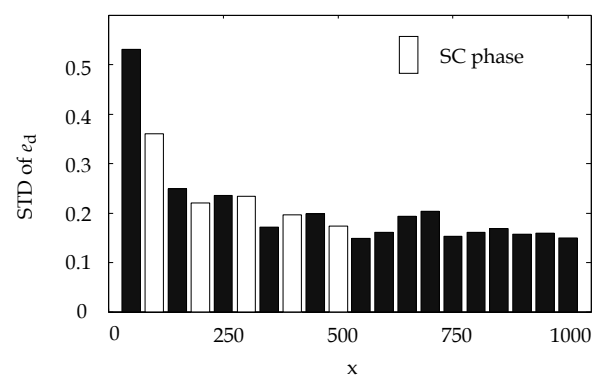

(f) STD of following error $e_{\mathrm{d}}$ with SC

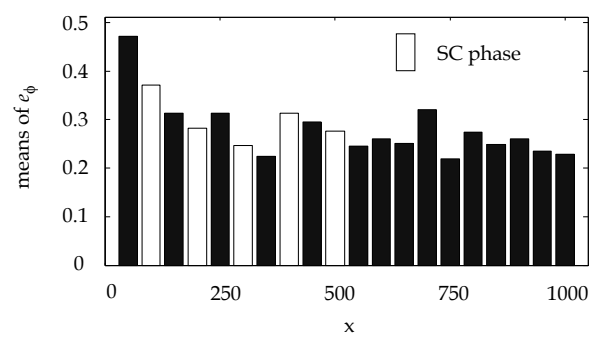

(g) means of trotational error $e_{\phi}$ with assist

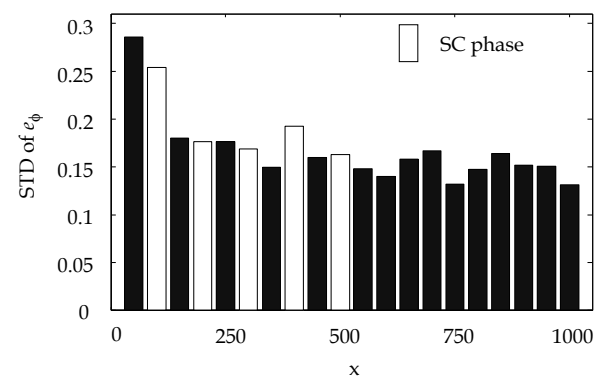

(h) STD of rotational error $e_{\phi}$ with SC

Fig. 7. Experimental results: means and STD of the evaluation value $e_{\mathrm{d}}$ and $e_{\phi}$ with/without the Subliminal Calibration (SC). 


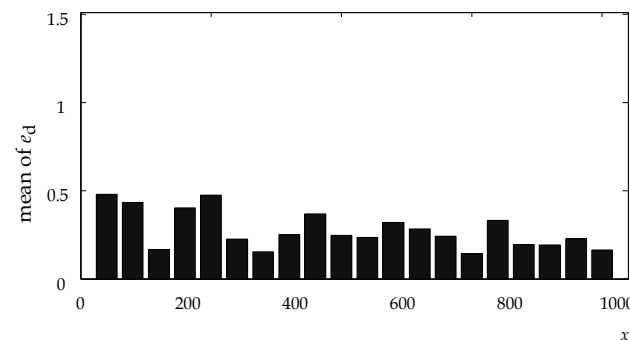

(a) following error without SC: skilled participant

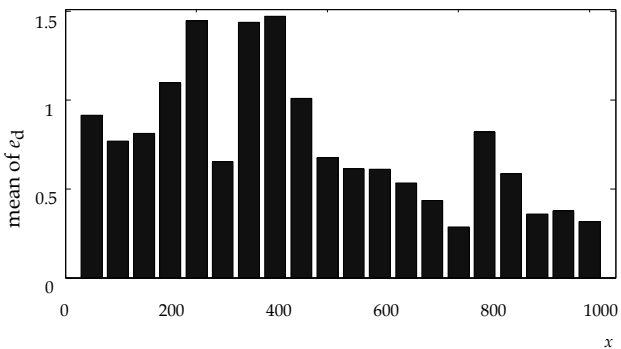

(b) following error without SC : unskilled participant

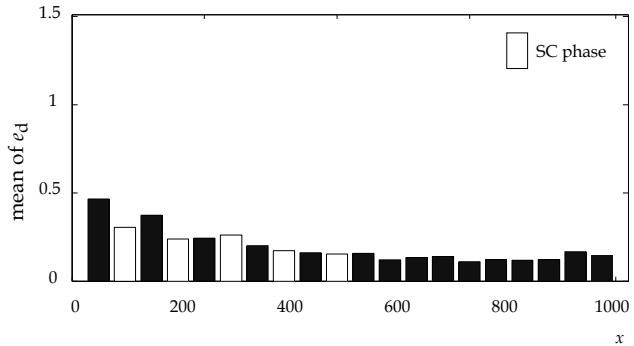

(b) following error with SC : skilled participant

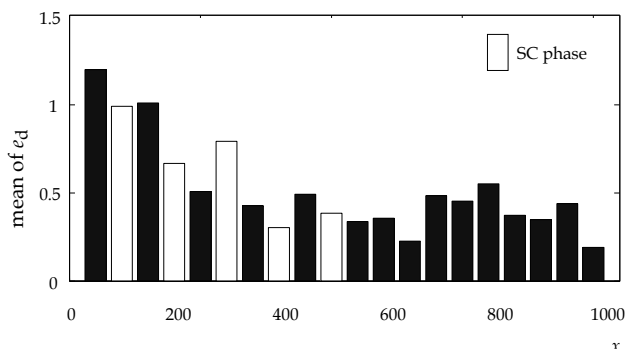

(d) following error with SC : unskilled participant

Fig. 8. Experiment results: following performance in skilled and unskilled operator.

\section{Conclusion}

Most of conventional assist techniques for human-machine systems relied on autonomous behaviour of the system with adding external force to command input, and thus on the subjectivity of system designers. These techniques, however, may work well only in a specific task a system designer assumed, and also may hinder the human initiative and learning ability.

Therefore, we proposed a calibration technique approaching the machine impedance to operator's internal model. This is expected to maximize the operability with which operator maneuver a machine at will with maintaining the initiative.

Human learning ability makes them feel uncomfortable in the face of variations in machine dynamics and it brings for the worse of operability. We set up a criterion on varying the operated machine impedance using JND as a perception criterion for varying stimulation. Variations in machine impedance within the limits of the criterion ensure calibration subliminally.

Our proposal requires estimation of an operator's internal model, so we conducted calibrations during a line tracking task in the experiment. The results show that all subjects improved line following accuracy without being aware of variations in operated vehicle impedance. This means that the vehicle dynamics approached the internal model, confirming that accuracy of $x>500$ was maintained even after the calibration stopped. In 
other words, our proposed calibration effectively customizes operated machine dynamics for individual subjects.

\section{Acknowledgement}

This research was partially supported by the Ministry of Education, Science, Sports and Culture, Grant-in-Aid for Scientific Research (A) (No. 20246071).

\section{References}

Brewer, B.; Fagan, M. ; Klatzky, R. \& Matsuoka, Y. (2005). Perceptual Limits for a Robotic Rehabilitation Environment Using Visual Feedback Distortion. IEEE Transactions on Neural Systems and Rehabilitation Engineering, Vol. 13, No. 1, pp.1-11

Cheng, G.; Zelinsky, A. \& Zelinsky, E. (1997). Supervised Autonomy: A Paradigm for Teleoperating Mobile Robots. Proceedings of the IEEE/RSJ International Conference on Intelligent Robots and Systems, pp. 973-980

Flanagan, J.; Nakano, E.; Imamizu, H.; Osu, R.; Yoshioka, T. \& Kawato, M. (1999). Composition and decomposition of internal models in motor learning under altered kinematic and dynamic environments. Journal of Neuroscience, 19:RC34

Forsyth, B. \& Maclean, K. (2006). Predictive Haptic Guidance: Intelligent User Assistance for the Control of Dynamic Tasks. IEEE Transactions on Visualization and Computer Graphics, Vol. 12, No. 1, pp.103-113

Furuta, K. (2003). Control of Pendulum: From Super Mechano-System to Human Adaptive Mechatronics. Proceedings of the 42nd IEEE Conference on Decision and Control, plenary talk, pp. 1498-1507

Horiguchi, Y. \& Sawaragi, T. (1999). Human-Robot Collaboration within a Virtual Space Using Behavioral Task-Morphologies. Proceedings of International Conference of IEEE System, Man and Cybernetics, Vol. 1, pp.756-761

Igarashi, H.; Takeya, A.; Kubo, Y.; Suzuki, S.; Harashima, F. \& Kakikura, M. (2005). Human Adaptive GUI Design for Teleoperation System. Proceedings of the 31st Annual Conference of the IEEEE Industrial Electronics Society, pp.1973-1978

Igarashi, H.; Takeya, A.; Harashima, F. \& Kakikura, M. (2006). Human Adaptive Assist Planning for Teleoperation. Proceedings of The 32nd Annual Conference of the IEEE Industrial Electronics Society, pp.4522-4527

Katsura, S. \& Ohnishi, K. (2007). "Acquisition and Analysis of Finger Motions by SkillPreservation System, IEEE Transactions on Industrial Electronics, Vol. 54, No. 6, pp.3353-3361.

Suzuki, S.; Harashima, F. \& Pan, Y. (2005). Assistant control design of HAM including human voluntary motion. Proceedings of the 2nd COE Workshop on Human Adaptive Mechatronics, pp.105-110

Teghtsoonian, R. (1971). On the Exponents in Sevens's Law and the Constant in Ehrman's Law. Psychological Review, Vol. 78, pp. 71-80

Wang, M. \& Liu, J. (2004). A Novel Teleoperation Paradigm for Human-Robot Interaction. Proceedings of IEEE International Conference on Robotics, Automation and Mechatronics, pp. 13-18 
Yamada, S. \& Yamaguchi, T. (2004). Training AIBO like a dog, Proceedings of the 13th International Workshop on Robot and Human Interactive Communication, pp.431-436

Zheng, X.; Tsuchiya, K.; Sawaragi, T.; Osuka, K.;Tsujita, K.; Horiguchi, Y. \& Aoi, S. (2004). Development of Human-Machine Interface in Disaster-Purposed Search Robot Systems That Serve as Surrogates for Human. Proceedings of IEEE International Conference on Robotics and Automation, pp. 225-230 


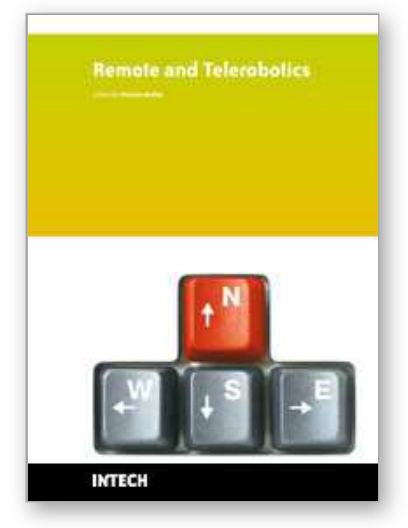

\author{
Remote and Telerobotics \\ Edited by Nicolas Mollet
}

ISBN 978-953-307-081-0

Hard cover, 220 pages

Publisher InTech

Published online 01, March, 2010

Published in print edition March, 2010

Any book which presents works about controlling distant robotics entities, namely the field of telerobotics, will propose advanced technics concerning time delay compensation, error handling, autonomous systems, secured and complex distant manipulations, etc. So does this new book, Remote and Telerobotics, which presents such state-of-the-art advanced solutions, allowing for instance to develop an open low-cost Robotics platform or to use very efficient prediction models to compensate latency. This edition is organized around eleven high-level chapters, presenting international research works coming from Japan, Korea, France, Italy, Spain, Greece and Netherlands.

\title{
How to reference
}

In order to correctly reference this scholarly work, feel free to copy and paste the following:

Hiroshi Igarashi (2010). Subliminal Calibration for Machine Operation, Remote and Telerobotics, Nicolas Mollet (Ed.), ISBN: 978-953-307-081-0, InTech, Available from: http://www.intechopen.com/books/remote-andtelerobotics/subliminal-calibration-for-machine-operation

\section{INTECH}

open science | open minds

\section{InTech Europe}

University Campus STeP Ri

Slavka Krautzeka 83/A

51000 Rijeka, Croatia

Phone: +385 (51) 770447

Fax: +385 (51) 686166

www.intechopen.com

\section{InTech China}

Unit 405, Office Block, Hotel Equatorial Shanghai

No.65, Yan An Road (West), Shanghai, 200040, China

中国上海市延安西路 65 号上海国际贵都大饭店办公楼 405 单元

Phone: +86-21-62489820

Fax: +86-21-62489821 
(C) 2010 The Author(s). Licensee IntechOpen. This chapter is distributed under the terms of the Creative Commons Attribution-NonCommercialShareAlike-3.0 License, which permits use, distribution and reproduction for non-commercial purposes, provided the original is properly cited and derivative works building on this content are distributed under the same license. 